\title{
MORFOLOGIA GONADAL E BIOLOGIA REPRODUTIVA DE FÊMEAS DE Callinectes ornatus (CRUSTACEA: DECAPODA) NO LITORAL SUL CAPIXABA
}

\author{
Renzo Gonçalves Tavares ${ }^{1}$ \\ Jamily Almeida Fontoura² \\ Tugstênio Lima de Souza ${ }^{3}$ \\ Adriane Araújo Braga ${ }^{4}$ \\ Erika Takagi Nunes ${ }^{5}$
}

Resumo: Callinectes ornatus é uma espécie de siri-azul que é utilizado como um importante recurso pesqueiro. O objetivo deste trabalho foi classificar a morfologia macroscópica da gônada, os estágios de desenvolvimento e aspectos acerca de sua biologia reprodutiva. Para tal, os animais foram coletados na região sul do litoral capixaba com o auxílio de uma embarcação e rede de arrasto. As fêmeas foram classificadas nos estágios: imaturo (IM), rudimentar (RU), em desenvolvimento (ED) e desenvolvido (ED). Quanto aos aspectos ecológicos foi observada uma predominância de fêmeas imaturas em todos os pontos amostrados, na estação seca e chuvosa. Já as desenvolvidas apareceram em maior quantidade apenas na estação seca. Sendo assim, de acordo com os dados obtidos foi possível caracterizar o ciclo reprodutivo desta espécie como sazonal-contínua.

Palavras-chave: Reprodução; Siri-azul; Ecologia; Gônada; Caracterização macroscópica.

\footnotetext{
${ }^{1}$ Ciências Biológicas/Universidade Federal do Espírito Santo, Brasil. E-mail: renzo_tavares@hotmail.com.

2 Ciências Biológicas/Universidade Federal do Espírito Santo, Brasil. E-mail: jamilyfontoura@gmail.com.

3 Ciências Biológicas/Universidade Federal do Espírito Santo, Brasil. E-mail: tugstenio94@hotmail.com.

${ }^{4}$ Ciências Biológicas/Universidade Federal do Espírito Santo, Brasil. E-mail: dricrab@yahoo.com.br.

${ }^{5}$ Ciências Biológicas/Universidade Federal do Espírito Santo, Brasil. E-mail: erikatnunes@yahoo.com.br.
} 